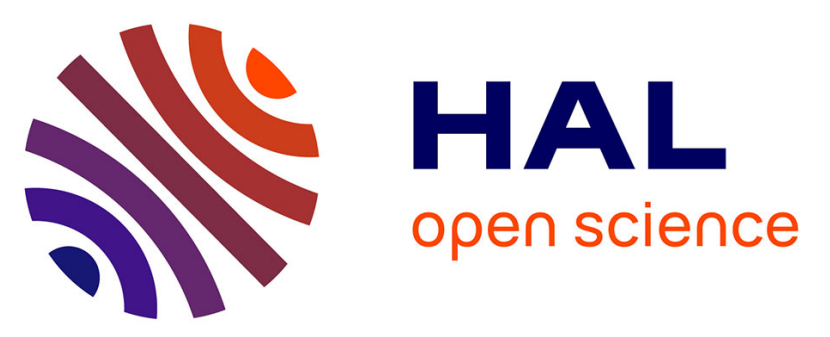

\title{
Utilisation du logiciel PELICAN pour la saisie et l'export de comptes rendus standardisés dans les tumeurs du système nerveux central : application aux méningiomes
}

Clémence Yguel, Dominique Clauzon, Stéphanie Lacomme, Sandra Lomazzi, Emilie Lardenois, Celso Pouget, Luc Taillandier, Fabien Rech, Valérie Rigau, Jean-Michel Vignaud, et al.

\section{- To cite this version:}

Clémence Yguel, Dominique Clauzon, Stéphanie Lacomme, Sandra Lomazzi, Emilie Lardenois, et al.. Utilisation du logiciel PELICAN pour la saisie et l'export de comptes rendus standardisés dans les tumeurs du système nerveux central : application aux méningiomes. Annales de Pathologie, 2019, 39 (6), pp.414-424. 10.1016/j.annpat.2019.01.016 . hal-02419393

\author{
HAL Id: hal-02419393 \\ https://hal.science/hal-02419393
}

Submitted on 21 Dec 2021

HAL is a multi-disciplinary open access archive for the deposit and dissemination of scientific research documents, whether they are published or not. The documents may come from teaching and research institutions in France or abroad, or from public or private research centers.
L'archive ouverte pluridisciplinaire HAL, est destinée au dépôt et à la diffusion de documents scientifiques de niveau recherche, publiés ou non, émanant des établissements d'enseignement et de recherche français ou étrangers, des laboratoires publics ou privés.

\section{(ㄷ)(1) $\$$}

Distributed under a Creative Commons Attribution - NonCommerciall 4.0 International 


\section{Utilisation du logiciel PELICAN pour la saisie et l'export de comptes-rendus standardisés dans les tumeurs du système nerveux central : application aux méningiomes}

Use of the PELICAN software for the creation and export of standardized pathology reports in central nervous system tumors: example of meningiomas

Clémence YGUEL (1), Dominique CLAUZON (2), Stéphanie LACOMME (3), Sandra LOMAZZI (3), Emilie LARDENOIS (1), Celso POUGET (1), Luc TAILLANDIER (4), Fabien RECH (5,6), Valérie RIGAU (7), Jean-Michel VIGNAUD $(1,3,8)$, Luc BAUCHET (9), Guillaume GAUCHOTTE $(1,3,8)$

(1) Service d'Anatomie et Cytologie Pathologiques, Hôpital Central, CHRU de Nancy, 54000 NANCY, France

(2) Ingénieur consultant en solutions et développements informatiques, 54000 Nancy, France

(3) Centre de Ressources Biologiques BB-0033-00035, CHRU de Nancy, 54000 Nancy, France

(4) Service de Neurologie, Hôpital Central, CHRU de Nancy, 54000 Nancy, France

(5) Service de Neurochirurgie, Hôpital Central, CHRU de Nancy, 54000 Nancy, France

(6) Institut des Neurosciences, INSERM U1051, Montpellier, F-34091, France

(7) Service d'Anatomie et Cytologie Pathologiques, CHU de Montpellier, 34000 Montpelier, France

(8) INSERM U1256, équipe 3 MIGB, NGERE, Université de Lorraine, 54500 VANDOEUVRELES-NANCY, France

(9) Service de Neurochirurgie, CHU de Montpellier, 34000 Montpellier, France

\section{Auteur correspondant :}

Pr Guillaume GAUCHOTTE

Service d'Anatomie et Cytologie Pathologiques

Hôpital Central, CHRU de Nancy

29, avenue du Maréchal de Lattre de Tassigny

54000 Nancy, France

E-mail : g.gauchotte@chru-nancy.fr

Tel : +33383851351

Fax : +33 383851331

Mots clés : PELICAN, méningiome, compte-rendu standardisé, base de données, anatomie et cytologie pathologiques

Keywords : PELICAN, meningioma, standardized report, database, pathology 


\section{Résumé}

Introduction: L'application PELICAN («Partager Efficacement en Laboratoire les Informations des Comptes rendus ANatomopathologiques ») est un outil permettant de générer des comptes-rendus standardisés et de renseigner automatiquement une base de données, utilisée au CHRU de Nancy depuis 2014 en neuropathologie tumorale. L'objectif de cet article était d'illustrer l'utilisation de cette application pour les méningiomes, avec une première exploitation statistique.

Matériels et Méthodes : L'étude inclut les cas de méningiomes enregistrés dans l'application PELICAN jusqu'en juillet 2018. L'application PELICAN est un fichier Microsoft Excel comportant un logiciel écrit en Visual Basic pour Applications, utilisé par le pathologiste pour élaborer le compte-rendu. Les principales données cliniques ont été recueillies à partir de la fiche de recensement du Registre de l'Hérault. Le suivi était renseigné de façon systématique pour les méningiomes atypiques.

Résultats : 295 méningiomes ont été analysés dont 250 méningiomes de grade I, 42 méningiomes de grade II et 3 méningiomes de grade III. Dans les méningiomes de grade II, on constatait une proportion significativement plus élevée d'hommes $(p=0,002)$ et d'infiltration de la dure-mère $(\mathrm{p}<0,001)$, une augmentation significative de l'indice Ki-67 $(\mathrm{p}<0,0001)$ et une diminution significative de l'expression des récepteurs à la progestérone $(\mathrm{p}<0,001)$. Dans les méningiomes atypiques, un indice Ki-67 de plus de $20 \%$ était corrélé significativement à une survie sans progression plus courte $(\mathrm{p}=0,032)$.

Conclusion : L'application PELICAN est un outil d'utilisation simple, permettant de générer des comptes-rendus standardisés tout en alimentant une base de données, ouvrant de très intéressantes perspectives d'un point de vue épidémiologique et scientifique.

\section{Summary:}

Introduction: PELICAN ("Partager Efficacement en Laboratoire les Informations des Comptes-rendus ANatomopathologiques") is a software which generates standardized reports, and allows to automatically create a database. It has been used in central nervous system tumor pathology at the University Hospital of Nancy since 2014. The purpose of this article was to illustrate the use of this application for meningiomas, with a first statistical evaluation.

Materials and methods: The export of data included all cases of meningiomas recorded in the PELICAN application until July 2018. The PELICAN application is a Microsoft Excel file containing a software, written in Visual Basic for Applications, and used by the pathologist to create the report. The main clinical data were collected from the Hérault Register census form. Follow-up was systematically reported for atypical meningiomas.

Results: 295 meningiomas were analyzed, including 250 grade I meningiomas, 42 grade II meningiomas, and 3 grade III meningiomas. Grade II meningiomas were characterized by a significantly higher proportion of men $(\mathrm{p}=0.002)$ and dural infiltration $(\mathrm{p}<0.001)$, a significant 
increase in the Ki-67 index $(\mathrm{p}<0.0001)$, and a significant decrease in progesterone receptor expression $(\mathrm{p}<0.001)$. In atypical meningiomas, a Ki-67 index of more than $20 \%$ was significantly correlated with a shorter progression-free survival $(\mathrm{p}=0.032)$.

Conclusion: The PELICAN software is an easy-to-use tool that allows to generate standardized reports and feed a database, opening very interesting perspectives from an epidemiological and scientific point of view. 


\section{Introduction}

L'application PELICAN (« Partager Efficacement en Laboratoire les Informations des Comptes rendus ANatomopathologiques ») a été initiée en 2014 en neuropathologie dans le cadre d'un projet de l'école ARTEM (ARt, Technologie Et Management), dont l'objectif était la création et l'activation d'une base de données exhaustive des tumeurs du système nerveux central [1], avec le soutien de l'ANOCEF (Association des Neuro-Oncologues d'Expression Française) et du Registre des Tumeurs de l'Hérault. Ce projet répondait à plusieurs problèmes et demandes : l'absence de normalisation des données devant figurer dans les comptes rendus d'anatomopathologie, l'absence de mémorisation structurée des données des comptes rendus d'anatomopathologie, et la nécessité de ressaisie des informations pour les logiciels de tumorothèques et autres bases de données, comme en particulier pour le Recensement national histologique des Tumeurs Primitives du Système Nerveux Central (RnhTPSNC) du Registre des Tumeurs de l'Hérault, base nationale des tumeurs du système nerveux central. Depuis 2014, et jusqu'à la date de rédaction de cet article (juillet 2018), 886 tumeurs du système nerveux central ont été enregistrées dans l'application dont 295 méningiomes (33\%).

L'application PELICAN comporte différents formulaires spécifiques permettant la génération de comptes-rendus standardisés pour la très grande majorité des tumeurs du SNC. Pour les tumeurs répondant à la classification OMS 2016 des tumeurs du SNC [2], six types d'écrans spécifiques de saisie sont disponibles: gliomes diffus, gliomes circonscrits et tumeurs glio-neuronales, tumeurs embryonnaires, méningiomes, tumeurs épendymaires et plexuelles, et lymphomes. Par ailleurs, depuis quelques mois, l'application permet de générer des comptes-rendus standardisés pour les adénomes hypophysaires, en suivant la classification OMS 2017 des tumeurs endocrines [3]. Dans cet article, nous nous focaliserons sur l'interface dédiée aux méningiomes. Les méningiomes représentent entre 24 et $40 \%$ des tumeurs cérébrales primitives de l'adulte avec une incidence de 6 à 13/100 000 habitants/an, l'incidence variant selon l'origine ethnique [2,4]. Ils se développent dans l'espace sous-dural aux dépens des cellules arachnoïdiennes. Le pic d'incidence se situe autour de la sixième décennie avec une prédominance féminine (sex-ratio à 2:1) et ils sont rares chez l'enfant et l'adulte jeune. Ils sont généralement plus agressifs chez les hommes et en cas de tumeur radio-induite. La plupart des méningiomes sont sporadiques, mais peuvent s'intégrer dans le cadre de syndrome familiaux (ex. : syndrome de Li-Fraumeni) ou de la neurofibromatose de type 2. Quelques facteurs de risque ont été décrits, comme les radiations ionisantes, et les facteurs hormonaux [5]. La classification 2016 de l'Organisation Mondiale de la Santé (OMS) des tumeurs du système nerveux central classe les méningiomes en trois grades histopronostiques : les méningiomes à faible risque de grade I dits «bénins »; et les méningiomes à fort risque de récidive et/ou de comportement agressif : les méningiomes de grade II dits «atypiques » et les méningiomes de grade III dits «anaplasiques » [2]. Un méningiome de grade II se définit par la présence d'un critère majeur (invasion du parenchyme cérébral, présence de 4 mitoses ou plus pour 10 champs au fort grossissement [x400;0,16 mm² par champ]) ou d'au moins trois critères mineurs parmi les suivants : une densité cellulaire augmentée, des petites cellules à rapport nucléo-cytoplasmique augmenté, des nucléoles proéminents, une perte de l'architecture et des foyers de nécrose tumorale. Les sous-types 
chordoïde et à cellules claires sont par définition de grade II selon l'OMS. Un méningiome de grade III se définit soit par la présence de 20 mitoses ou plus pour 10 champs au fort grossissement (x400;0,16 $\mathrm{mm}^{2}$ par champ) ou un aspect anaplasique pouvant évoquer un carcinome, un mélanome ou un sarcome de haut grade. Du fait de leur comportement agressif, les sous-types rhabdoïde et papillaire sont par définition de grade III de l'OMS. La survie à 5 ans est à plus de $90 \%$ pour les méningiomes de grade I, entre 60 et $80 \%$ pour les méningiomes de grade II et entre 30 et $50 \%$ pour les méningiomes de grade III. Le traitement repose essentiellement sur la chirurgie qui doit être la plus complète possible, la récidive tumorale étant un problème fréquent [6]. Une radiothérapie peut être proposée pour les méningiomes inopérables, évolutifs ou récidivants, ou en traitement complémentaire post-opératoire [7].

L'objectif de cet article est d'illustrer l'utilisation de l'application PELICAN pour les méningiomes. Nous détaillerons tout d'abord l'interface de saisie du compte-rendu standardisé, puis nous effectuerons une première exploitation statistique des données.

\section{Matériels et Méthodes}

\section{Cas étudiés}

Les cas analysés étaient tous les méningiomes enregistrés dans l'application PELICAN depuis sa création en octobre 2014 jusqu'au 5 juillet 2018, dans le service d'Anatomie et Cytologie Pathologiques du CHRU de Nancy, l'utilisation de l'application étant quasi-systématique pour le diagnostic des méningiomes depuis fin 2014.

\section{Infrastructure de l'application et élaboration de l'interface de saisie}

L'application PELICAN est un fichier Excel comportant un logiciel spécifiquement développé pour les laboratoires, écrit en Visual Basic pour Applications (VBA), ne nécessitant pas d'autre suite logicielle que Microsoft Office. Il gère en complète autonomie une base de données spécifique partageable en mode multi-utilisateurs sur le réseau local du CHRU de Nancy. L'exportation des données se fait par copie du compte rendu sous forme de texte mis en forme et collage dans le logiciel métier du laboratoire (DIAMIC).

Le premier écran de saisie est commun à toutes les tumeurs du SNC. L'interface de saisie spécifique aux méningiomes et autres tumeurs méningées a été mise au point en se basant sur la «fiche relecture des méningiomes, tumeurs fibreuses solitaires, hémangiopéricytomes » proposée en 2014 dans le cadre du RENOP (Réseau national de NeuroOncologie Pathologique). L'onglet dédié à l'immunohistochimie est inspiré des recommandations rédigées en 2016 par les Professeurs G. Gauchotte et F. Labrousse sur les prérequis de techniques immunohistochimiques et/ou moléculaires à réaliser avant toute demande de relecture de méningiomes dans le cadre du RENOP. 
Les données nécessaires à l'élaboration du compte-rendu standardisé peuvent être renseignées directement par le pathologiste à l'aide de champs de formulaire avec des cases à cocher. Il est également possible si nécessaire de rajouter du texte libre, pouvant être saisi directement par le pathologiste, ou par le secrétariat après dictée vocale.

En complément des données servant à élaborer le compte-rendu, il est possible de saisir des données cliniques et radiologiques, via des écrans spécifiques de saisie intitulés «contexte» et «topographie». Ces données sont issues de la feuille de recensement du RnhTPSNC, habituellement remplie par les chirurgiens dans le cadre de ce recensement. Secondairement, un onglet «Informations cliniques» a été introduit, comportant en particulier les données de suivi. Dans notre laboratoire, les données cliniques sont renseignées par un ingénieur du Centre de Ressources Biologiques (CRB).

La codification des lésions est double, se basant à la fois sur les codes SNOMED (Systematized Nomenclature of MEDicine) issus de la classification OMS 2016 des tumeurs du SNC et les codes élaborés par l'ADICAP (Association pour le Développement de l'Informatique en Cytologie et en Anatomie Pathologiques) en novembre 2009, version 5.04 [8].

\section{Export des données}

Les informations statistiques globales ont été extraites du logiciel à partir de l'onglet dédié «statistiques générales ». L'export des données enregistrées a été effectué par le biais de requêtes multicritères dans la partie «analyse des données de la base PELICAN ». Les requêtes ont porté sur l'ensemble des données anatomopathologiques relatives aux méningiomes (groupe 110), et de l'ensemble des données clinico-radiologiques, chirurgicales et données issues des feuilles de recensement. L'exportation des données sélectionnées se fait directement à partir de l'application PELICAN sous forme de table Excel.

\section{Réglementation}

L'application PELICAN s'inscrit dans le cadre du respect de la confidentialité des informations conformément à l'article 34 de la loi «Informatique et Libertés » du 6 janvier 1978, et par le respect des référentiels de sécurité et d'interopérabilité prévu par la loi (article L 1111-8 alinéa 4 du Code de la santé publique) et définis par l'ASIP-Santé, en conformité avec le cadre juridique relatif à l'hébergement des données de santé à caractère personnel prévu par la loi (article L 1111-8 du Code de la santé publique). Cette application est conforme à la réglementation européenne (Règlement Général de Protection des Données, 25 mai 2018). 
L'analyse statistique a été effectuée à l'aide du logiciel GraphPad PRISM. La comparaison de la répartition entre les groupes (méningiomes de grade I vs. II) des variables qualitatives a fait l'objet d'un test du Khi² ou d'un test de Fisher (en cas d'effectifs < 5), et des données quantitatives d'un test de Mann-Whitney-Wilcoxon. Une p-value inférieure à 0,05 est considérée comme statistiquement significative.

\section{Résultats}

\section{Description de l'application PELICAN}

L'écran d'accueil est subdivisé en plusieurs zones permettant d'accéder à diverses fonctionnalités de l'application (Figure $1 \mathrm{~A}$ ). A gauche de l'écran, la bibliothèque présente les différents dossiers qui sont stockés dans la base, en cours de rédaction, validés dont les données cliniques sont à compléter, ou terminés. Un nouveau ficher (dossier PELICAN) peut être généré à partir de cette bibliothèque. Les données du compte rendu d'anatomopathologie $(\mathrm{CR}-\mathrm{ACP})$ sont recueillies dans la partie «compte rendu d'examen », les données relatives à la feuille de recensement sont remplies dans les deux onglets "contexte » et «topographie », et les données cliniques et de suivi sont à compléter dans l'onglet «informations cliniques » (Figure $1 \mathrm{~A}$ ).

Les données du CR-ACP sont recueillies dans un écran de saisie d'informations communes à l'ensemble des tumeurs (numéro d'examen, date, identification des praticiens concernés, congélation, inclusion en totalité, qualité du prélèvement, etc.) (Figure $1 \mathrm{~B}$ ), et deux écrans spécifiques («saisie des informations spécifiques au groupe tumoral » et «saisie du diagnostic et de la conclusion ») qui dépendent du type tumoral choisi par le médecin. Des champs libres de macroscopie et microscopie peuvent être remplis si nécessaire, en complément des informations structurées (Figure $1 \mathrm{~B}$ ).

L'écran «saisie des informations spécifiques » comprend deux onglets : « examen du prélèvement » et «analyse ». Ce premier onglet est divisé en différents paragraphes : siège de la tumeur, architectures et contingents cellulaires d'intérêt pronostiques (en particulier, aspect chordoïde, à cellules claires, rhabdoïde ou papillaire), critères histo-pronostiques majeurs (mitoses, invasion du parenchyme cérébral), mineurs (hypercellularité, nécrose, petites cellules, nucléoles proéminents, perte de l'architecture tumorale) et autres (Figure 2 A). Dans les autres critères, l'hyperplasie vasculaire et la prolifération endothélio-capillaire ont été rajoutées récemment, suite à une étude que nous avons menée en 2016 [9]. Les données non renseignées, notamment lorsqu'elles n'apportent pas d'élément pertinent, ou si non applicables, sont exclues du compte-rendu final, afin de ne pas alourdir inutilement ce dernier. L'écran «analyse » regroupe l'immunohistochimie (Figure $2 \mathrm{~B}$ ) et la biologie moléculaire (LOH 22q12.2, mutation NF2, fusion NAB2:STAT6, autre). Pour chaque anticorps, il faut préciser si le marquage est positif ou négatif et le pourcentage de cellules marquées s'il est positif. Les éventuelles données de biologie moléculaire peuvent être remplies dans un second temps, compte tenu des délais techniques. 
L'onglet «saisie du diagnostic et de la conclusion » (Figure $2 \mathrm{C}$ ) permet de définir le diagnostic retenu parmi les entités présentes dans le groupe tumoral choisi (ex. : méningiome méningothélial, entité du groupe méningiome), de préciser le grade histo-pronostique issu de la classification OMS 2016 des tumeurs du système nerveux central [2]. Les codes ADICAP et SNOMED sont automatiquement renseignés. La conclusion doit être complétée dans le texte libre situé en bas de l'écran. En revenant à l'écran d'accueil, le compte-rendu standardisé mis en forme est généré en cliquant sur «copier le compte-rendu », celui-ci pouvant alors être collé dans le logiciel métier du laboratoire.

\section{Données cliniques}

Deux cent quatre-vingt-quinze méningiomes ont été recensés avec l'application jusqu'en juillet 2018, soit 33\% des tumeurs du système nerveux central enregistrées dans l'application PELICAN, dont 250 méningiomes de grade I (85\%), 42 méningiomes de grade II (14\%) et 3 méningiomes de grade III (1\%) (tableau 1). La moyenne d'âge au diagnostic était de 58 ans. Soixante-seize pourcents atteignaient des femmes. La proportion d'homme augmentait significativement dans les méningiomes de grade II par rapport aux méningiomes de grade I (42\% vs. $24 \%, \mathrm{p}=0,002)$.

Les symptômes les plus souvent rencontrés étaient les troubles des fonctions supérieures, les céphalées et l'épilepsie, sans différence significative selon le grade. Le score OMS était toujours de 0 . Le délai entre le début des symptômes et la chirurgie était généralement inférieur à 12 mois que ce soit dans les méningiomes de grade I ou de grade II (tableau 2). Les méningiomes siégeaient principalement au niveau de la convexité (42\%), puis de la base du crâne (30\%).

Les données thérapeutiques et de suivi renseignées intéressaient quasi-exclusivement les méningiomes de grade II et montraient une récidive tumorale pour $35 \%$ de ces derniers. Aucune métastase et aucun décès n'ont été rapportés. Seize pourcents des patients atteintes de méningiome de grade II ont bénéficié d'une embolisation et $73 \%$ d'un traitement adjuvant: $61 \%$ de radiothérapie, $3 \%$ de radio-chimiothérapie et $12.5 \%$ d'autre traitement.

\section{Données histopathologiques}

Les méningiomes étaient à $40 \%$ de type méningothélial, $12 \%$ de type fibroblastique, 16\% de type transitionnel (mixte), 6\% de type psammomateux et 6\% d'architecture microkystique. Les autres types architecturaux représentaient chacun moins de $2 \%$ (Tableau 1). Des aspects chordoïdes, à cellules claires, rhabdoïdes ou papillaires étaient observées dans respectivement $1 \%, 3 \%, 1 \%$ et $1 \%$ des cas, dans la grande majorité des cas de façon focale. Seul un méningiome de grade II présentait une architecture chordoïde de façon diffuse $(0.3 \%)$. 
Des critères histo-pronostiques péjoratifs mineurs étaient retrouvés dans quelques méningiomes de grade I (de $6 \%$ à $12 \%$ selon les critères), alors que $71 \%$ des méningiomes de grade II présentaient au moins 3 critères sur 5. L'hyperplasie endothéliale était observée dans $12 \%$ des méningiomes de grade I et $75 \%$ des méningiomes de grade II $(\mathrm{p}=0.002)$. La prolifération endothélio-capillaire était retrouvée dans $8 \%$ des méningiomes de grade I et 50 $\%$ des méningiomes de grade II avec une p-value à 0.03 (Tableau 2). La figure 3 illustre l'aspect histologique d'une prolifération endothélio-capillaire et d'une hyperplasie endothéliale dans un cas de méningiome atypique (Figure 3 A et Figure 3 B).

L'indice mitotique moyen était de 0.7 mitoses $/ 1.6 \mathrm{~mm}^{2}$ pour les méningiomes de grade I et de 4.5 mitoses $/ 1.6 \mathrm{~mm}^{2}$ pour les méningiomes de grade II. $50 \%$ des méningiomes de grade II avaient une activité mitotique inférieure à 4 mitoses $/ 1.6 \mathrm{~mm}^{2}$. Tous les méningiomes de grade III présentaient plus de 20 mitoses $/ 1.6 \mathrm{~mm}^{2}$.

Une invasion du parenchyme cérébral était présente dans $10 \%$ des cas de méningiome de grade II et $33 \%$ des méningiomes de grade III. La dure-mère était infiltrée dans $31 \%$ des cas de méningiomes de grade I contre $64 \%$ des méningiomes de grade II ( $p<0,001)$. Une infiltration osseuse était présente dans $7 \%$ des méningiomes de grade I contre $13 \%$ des méningiomes de grade II $(\mathrm{p}=0,32)$.

Il n'y avait pas de différence significative en terme de survie sans progression entre les méningiomes de grade II ayant un indice mitotique inférieur à 4 mitoses $/ 1,6 \mathrm{~mm}^{2}$ et ceux ayant un indice mitotique supérieur à 4 mitoses $/ 1,6 \mathrm{~mm}^{2}(\mathrm{p}=0.274)$.

\section{Données immunohistochimiques}

L'indice de prolifération évalué à l'aide de l'anticorps anti-Ki-67 (MIB1) était significativement plus élevé dans les méningiomes de grade II, avec un indice Ki-67 moyen de $5 \%$ pour les méningiomes de grade I contre $16 \%$ pour les méningiomes de grade II ( $\mathrm{p}=$ 0,001) (Figure $3 \mathrm{C}$ et Figure $3 \mathrm{D}$, Tableau 2). La survie sans progression était significativement plus courte pour les méningiomes atypiques présentant un indice de prolifération Ki-67 supérieur ou égal à 20\% ( $\mathrm{p}=0,032$; Log-Rank) que pour les méningiomes atypiques avec indice prolifératif plus faible.

Les anticorps les plus usités, en dehors de Ki-67, étaient ceux dirigés contre les récepteurs à la progestérone (95\%), SSTR2 (46\%), l'EMA (16.5\%) et STAT6 (18\%). Selon les sous-types histologiques, le panel d'anticorps utilisé était différent (Tableau 3). Tous stades confondus, l'EMA était positive dans $90 \%$ des cas et SSTR2 dans $98 \%$ des cas. Les récepteurs à la progestérone étaient exprimés dans $96 \%$ des méningiomes de grade I, $87 \%$ des méningiomes de grade II et $33 \%$ des méningiomes de grade III. La moyenne de cellules marquées était de $48 \%$ pour les méningiomes de grade I (Figure $3 \mathrm{E}$ ) et de $28 \%$ pour les méningiomes de grade II (Figure 3 F) (p < 0.001) (Tableau 2). 


\section{Discussion}

L'application PELICAN a été initiée en 2014 en neuropathologie dans le cadre du projet ARTEM, dont l'objectif était la création et l'activation d'une base de données exhaustive des tumeurs du SNC. A ce jour, environ 900 tumeurs primitives du SNC y sont enregistrées. Il s'agit d'une application originale, facilement utilisable en routine, constituant dans notre expérience un gain de temps par rapport aux comptes-rendus classiques, et ne nécessitant pas d'autre suite logicielle que Microsoft Office. Elle est un apport considérable d'un point de vue épidémiologique et scientifique avec la possibilité d'enregistrer, de façon couplée aux données des comptes rendus anatomopathologiques, des renseignements cliniques, y compris les données de survie.

Nous avons effectué dans cet article la première exploitation statistique des données enregistrées depuis sa création en 2014. Cette analyse a portée sur 250 méningiomes de grade I, 42 méningiomes de grade II et 3 méningiomes de grade III. Ces données, exportées sous format Excel, ont été importées dans un logiciel de biostatistiques et ont permis de constater qu'il n'y avait pas de différence significative entre les méningiomes de grade I et ceux de grade II pour la symptomatologie au diagnostic, le score OMS et le délai entre l'apparition des symptômes et la prise en charge chirurgicale.

Concernant les méningiomes de grade I, les sous-types histologiques prédominant étaient le méningiome méningothélial, suivi du méningiome transitionnel puis du méningiome fibroblastique, de façon globalement similaire aux données de la littérature, en particulier issues du recensement national [10]. Des critères histo-pronostiques péjoratifs mineurs étaient retrouvés dans 6 à 12\% des méningiomes de grade I et entre 50 et $71 \%$ des méningiomes de grade II. Aucun méningiome de grade I ne présentait plus de 3 critères sur 5 (par définition), contre $71 \%$ des méningiomes de grade II. Les $29 \%$ de méningiomes de grade II ne présentant pas au moins 3 de ces critères mineurs étaient classés en tumeurs de grade II sur la base d'un sous-type chordoïde dans un cas, d'une invasion isolée du parenchyme cérébral dans un cas, et du fait d'un indice mitotique d'au moins 4 mitoses pour $1.6 \mathrm{~mm}^{2}$ pour tous les autres cas. L'hyperplasie endothéliale était présente dans 12\% des méningiomes de grade I contre 75\% des méningiomes de grade II $(\mathrm{p}=0.002)$. Ce critère semble ainsi être un critère histopronostique péjoratif pertinent, en accord avec une étude que nous avons publiée récemment [9].

D'un point de vue immunohistochimique, l'indice de prolifération était en moyenne de $5 \%$ pour les méningiomes de grade I et de $16 \%$ pour les méningiomes de grade II (p-value < 0.0001). Dans les méningiomes atypiques, malgré le nombre réduit de cas et la relativement faible durée du suivi clinique, nous avons constaté une corrélation inverse entre l'indice de prolifération Ki-67 et la survie sans progression ( $\mathrm{p}=0.032)$, comme cela a été rapporté dans d'autres études [11-13]. Par ailleurs, les récepteurs à la progestérone étaient positifs dans 96 $\%$ des méningiomes de grade I et $87 \%$ des méningiomes de grade II, avec un pourcentage moyen de cellules marquées de respectivement $48 \%$ et $28 \%$ (p<0.001), en accord avec les données de la littérature [14-16]. 
Ces premières exploitations statistiques permettent d'illustrer l'intérêt de la normalisation des comptes-rendus et de l'export des données. L'intégration progressive des données de suivi permettra d'évaluer facilement la valeur pronostique de différentes données histologiques ou moléculaires. Actuellement, seul le suivi des méningiomes atypiques est renseigné de façon exhaustive, avec cependant un recul clinique encore limité. Cette application pourra par ailleurs permettre de réaliser plus facilement des études sur des sousgroupes particuliers de méningiomes, en sélectionnant les cas selon la présence ou l'absence de critères histologiques ou immunohistochimiques d'intérêt. Etendre cet outil à d'autres centres permettrait bien sûr d'en renforcer l'intérêt, notamment en augmentant la puissance statistique. L'implication d'autres laboratoires avec un usage mutualisé nécessite cependant que chacun s'engage à respecter une charte d'utilisation, concernant notamment le partage des données.

Concernant les perspectives d'évolution de l'application, il est prévu dans les prochaines versions d'intégrer à l'application un outil permettant de générer des courbes de Kaplan-Meier couplées à des tests de survie (Log-Rank) ou d'autres tests statistiques, ce volet statistique étant actuellement en phase de développement. Par ailleurs, cette application pourra être dans l'avenir étendue à d'autres types de tumeur, en dehors du SNC. Par exemple, depuis le premier trimestre 2018, nous utilisons l'application PELICAN pour les pièces d'exérèse de tumeurs pulmonaires.

En conclusion, l'application PELICAN est un outil d'utilisation simple, permettant de générer des comptes rendus standardisés, tout en alimentant une base de données facilement exploitable sur le plan statistique, ouvrant de très intéressantes perspectives d'un point de vue épidémiologique et scientifique. Ce logiciel s'intègre de plus parfaitement dans le cadre politique actuel d'harmonisation des pratiques par la standardisation des comptes-rendus, tel que recommandé par les derniers Plans Cancer, et répond aux différentes normes actuelles en terme d'interopérabilité.

\section{Remerciements}

Les auteurs remercient l'ANOCEF, NENO (Nord Est Neuro-Oncologie) et M. Guy Mangin pour leur aide et leur soutien dans le développement initial du projet PELICAN ; le Centre de Ressources Biologiques Lorrain (CHRU de Nancy) pour son soutien financier.

\section{Déclaration de liens d'intérêts}

D.C. déclare avoir reçu une rémunération pour l'élaboration du logiciel PELICAN. Les autres auteurs n'ont pas de lien d'intérêt à déclarer.

\section{Références}


1) Verger L. CHRU de Nancy : la NENOBase pose sa 1ère pierre virtuelle, recherche clinique et innovation, CHRU Nancy, juillet 2015. Consultable sur le site http://recherche-innovation.chu-nancy.fr/publications/articles-archives/chru-denancyla-nenobase-pose-sa-1ere-pierre-virtuelle

2) Louis DN, Ohgaki H, Wiestler OD, Cavenee WK, Ellison DW, Figarella-Branger D, et al. WHO Classification of Tumours of the Central Nervous System. $4^{\text {th }}$ Edition, IARC, Lyon 2016:232-45

3) Lloyd RV, Osamura RY, Klöppel G and Rosai J. WHO Classification of Tumours of Endocrine Organs. $4^{\text {th }}$ Edition, IARC, Lyon 2017:11-64

4) Burger PC and Scheithauer BW. Tumors of the Central Nervous System. AFIP Atlas of Tumor Pathology. $4^{\text {th }}$ Edition. 2007;7:331-57

5) Shibuya M. Pathology and molecular genetics of meningiomas: recent advances. Neurol Med Chir (Tokyo) 2015;55:14-27

6) Simpson D. The recurrence of intracranial meningiomas after surgical treatment. J Neurol Neurosurg Psychiatry 1957;20:22-39

7) Goldbrunner R, Minniti G, Preusser M, Jenkinson MD, Sallabanda K, Houdart E, et al. EANO guidelines for the diagnosis and treatment of meningiomas. Lancet Oncol 2016;17:e383-91.

8) Thesaurus de la classification ADICAP, index raisonné des lésions, version 5.04, ADICAP, 2009. Consultable sur le site http://medphar.univ-poitiers.fr/registrecancers-poitou-charentes/documents_registre/adicap_version5_4_1_2009.pdf

9) Ling C, Pouget C, Rech F, Pflaum R, Treffel M, Bielle F, et al. Endothelial hyperplasia and microvascular proliferation in meningiomas are correlated with higher histological grade and shorter progression free survival. J Neuropathol Exp Neurol 2016;75:1160-1170

10) Zouaoui S, Darlix A, Rigau V, Mathieu-Daudé H, Bauchet F, Bessaoud F, et al.; French Brain Tumor DataBase (FBTDB) Participants and Investigators; with the participation of the Société française de neurochirurgie (SFNC); Club de neurooncologie de la SFNC; Société française de neuropathologie (SFNP); Association des neuro-oncologues d'expression française (ANOCEF). Descriptive epidemiology of 13,038 newly diagnosed and histologically confirmed meningiomas in France: 20062010. Neurochirurgie 2018;64:15-21.

11) Nanda A, Bir SC, Konar S, Maiti T, Kalakoti P, Jacobsohn JA, et al.Outcome of resection of WHO Grade II meningioma and correlation of pathological and radiological predictive factors for recurrence. J Clin Neurosci 2016;31:112-21

12) Champeaux C and Dunn L. World Health Organization Grade II Meningioma: A 10Year Retrospective Study for Recurrence and Prognostic Factor Assessment. World Neurosurg 2016;89:180-6

13) Gauchotte G, Vigouroux C, Rech F, Battaglia-Hsu SF, Soudant M, Pinelli C and Civit T. Expression of minichromosome maintenance MCM6 protein in meningiomas is strongly correlated with histologic grade and clinical outcome. Am J Surg Pathol 2012 Feb;36:283-91 
14) Omulecka A, Papierz W, Nawrocka-Kunecka A and Lewy-Trenda I. Immunohistochemical expression of progesterone and estrogen receptors in meningiomas. Folia Neuropathol 2006;44:111-5

15) Perry A, Cai DX, Scheithauer BW, Swanson PE, Lohse CM, Newsham IF, et al. Merlin, DAL-1, and progesterone receptor expression in clinicopathologic subsets of meningioma: a correlative immunohistochemical study of 175 cases. J Neuropathol Exp Neurol 2000;59:872-9

16) Hsu DW, Efird JT and Hedley-Whyte ET. Progesterone and estrogen receptors in meningiomas: prognostic considerations. J Neurosurg 1997;86:113-20 


\section{Légendes des figures et des tableaux}

Figure 1. Ecran d'accueil et écran de saisie d'informations communes aux différentes tumeurs du système nerveux central. Dans l'écran d'accueil (A), le compte-rendu peut être exporté à partir de l'onglet « copier le compte rendu » (flèche rouge). Les données relatives à la feuille de recensement sont remplies dans les onglets «contexte » et «topographie » (flèches noires); les données cliniques et de suivi sont complétées dans l'onglet « informations cliniques » (flèche bleue). A partir de l'écran d'informations communes (B), il est possible de saisir les informations générales, la présence de congélation, le groupe tumoral (flèche) et diverses informations. On the home screen and common data entry screen, which is shared for the different types of central nervous system tumors. In home screen (A), the report can be exported by clicking on "copy report" (red arrow). The census sheet data are completed in "context" and "topography" (black arrows); clinical and follow-up data are completed in the "clinical information" (blue arrow). From the common information screen $(B)$, we can detail general informations, frozen tissue avaibility, tumor group (arrow) and other various data.

Figure 2. Ecran des données spécifiques et écran «diagnostic et conclusion ». L'écran des données spécifiques comprend deux onglets : «examen du prélèvement » (A) et «analyse » (B). Le premier est divisé en plusieurs parties : siège de la tumeur, architectures et contingents cellulaires d'intérêt pronostique, critères histopronostiques majeurs et mineurs, et d'autres critères histopronostiques comme l'hyperplasie vasculaire et la prolifération endothéliocapillaire (flèche). Le second regroupe l'immunohistochimie et la biologie moléculaire. Les données non renseignées sont exclues du compte-rendu final. L'écran «diagnostic et conclusion» (C) permet de définir le diagnostic retenu et le grade histopronostique (flèche). Specific data entry screens and "diagnosis and conclusion» screen. The specific data screen has two tabs: "sample description" (A) and "analyses" (B). The first is divided into several parts: tumor site, architecture, architectures and cell components of prognostic value, major and minor histoprognostic criteria, and others histoprognostic criteria such as endothelial hyperplasia and microvascular proliferation (arrow). The second includes immunohistochemistry and molecular biology. Unfilled data are excluded from the final report. The screen "diagnosis and conclusion" $(C)$ allows to give the final diagnosis and the histoprognostic grade (arrow)

Figure 3. (A) Hyperplasie vasculaire (flèche) dans un cas de méningiome atypique (Hématoxyline, Eosine et Safran [HES] ; grossissement x100). (B) Prolifération endothélio-capillaire (flèche) dans un cas de méningiome atypique (HES ; x 200). (C) Indice de prolifération (Ki-67/MIB1) évalué à $1 \%$ dans un cas de méningiome de grade I (immunohistochimie [IHC] ; x200). (D) Indice de prolifération (Ki-67/MIB1) évalué à 50\% dans un cas de méningiome atypique (IHC; x200). (E) Expression des récepteurs à la progestérone dans $20 \%$ des cellules dans un cas de méningiome atypique (IHC; x40). (F) Expression des récepteurs à la progestérone dans $90 \%$ des cellules dans un cas de 
méningiome méningothélial de grade I (IHC; x 40). (A) Vascular hyperplasia (arrow) in a case of atypical meningioma (hematoxylin, eosin and saffron [HES]; magnification x 40). (B) Microvascular proliferation (arrow) in a case of atypical meningioma (HES; $x$ 40). (C) Proliferation index (Ki67/MIB1) at $1 \%$ in a case of grade I meningioma (immunohistochemistry [IHC]; x40). (D) Proliferation index (Ki67/MIB1) at 50\% in a case of atypical meningioma (IHC; 40 ). (E) Progesterone receptors expression in $20 \%$ of cells in a case of atypical meningioma (IHC; x40). (F) Progesterone receptors expression in $90 \%$ of cells in a case of grade I meningioma $(I H C ; x 40)$.

Tableau 1. Descriptif général des principales données concernant les méningiomes, extraites à partir de l'application PELICAN. General data about meningioma, after exportation from the PELICAN software.

Tableau 2. Comparaison des données cliniques, histologiques et immunohistochimiques entre les méningiomes de grade I et les méningiomes de grade II (test du $\mathrm{Khi}^{2}$ ou de Fisher pour les données qualitatives, test de Mann-Whitney-Wilcoxon pour les données quantitatives). Comparison of clinical, histological and immunohistochemical data between grade I and grade II meningiomas (Khi ${ }^{2}$ or Fisher test for qualitative variables, Mann-Whitney-Wilcoxon test for quantitative variables).

Tableau 3. Profil immunohistochimique des méningiomes selon leur sous-type histologique. Immunohistochemical profile of meningiomas according to histological subtype.

\section{Annexes}

Annexe 1. Exemple de compte rendu d'anatomopathologie standardisé généré par l'application PELICAN, pouvant être collé en mode mis en forme dans le logiciel métier du laboratoire. Example of standardized pathology report generated by the PELICAN application, which can be pasted in the laboratory software. 
A

\section{AN CEF}

Système ou Organe :

\section{Système nerveux central}

- Bibliothèque

\begin{tabular}{l|l} 
Tri par $\mathrm{n}^{\circ}$ & Tri par date \\
\hline
\end{tabular}

Pélican en cours de rédaction

$24 / 08 / 2018$

$20 / 08 / 2018$

$17 / 08 / 2018$

$13 / 08 / 2018$

$10 / 08 / 2018$

09/08/2018.

Nouveau Pélican

Pélican Validé, à compléter

\begin{tabular}{|lll|}
\hline $18 H$ & $06 / 06 / 2018$ & - \\
$18 H$ \\
$18 H$ \\
$18 H$ & $05 / 06 / 2018$ & - \\
$18 H$ & $05 / 06 / 2018$ & - \\
\hline $18 H$ & $05 / 06 / 2018$ & \\
$18 H$ & $04 / 06 / 2018$ & \\
Pélican Complété & $01 / 06 / 2018$ & \\
\hline $18 H$ & $01 / 06 / 2018$ & - \\
$18 H$ & & 422 \\
$18 H$ & $05 / 02 / 2018$ & - \\
$18 H$ & $16 / 02 / 2018$ & \\
$18 H$ & $16 / 02 / 2018$ & \\
$18 H$ & $12 / 02 / 2018$ & \\
$18 H$ & $12 / 02 / 2018$ & \\
\hline
\end{tabular}

$\ulcorner$ Masquer les Pélicans archivés

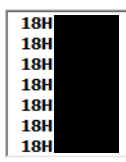

$22 / 08 / 2018$

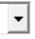

Commandes de pilotage du Pélican

Compte rendu d'examen

Copier le compte rendu

Valider le compte rendu

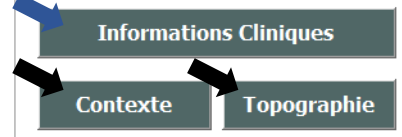

Informations Tumorothèque

Valider les compléments

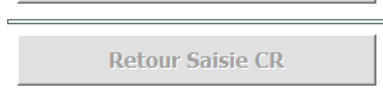

Compléter le Compte rendu

Relecture du Pélican
Position et Etat du Pélican

Date du prélèvement : 04/06/2018

Chirurgien :

Anatomopathologiste :

Pr Guillaume GAUCHOTTE

Diagnostic: Grade I

Méningiome méningothélia

\section{Version initiale Informations spécifiques saisies}

I. Informations communes à toutes les tumeurs.

W. Informations spécifiques à la tumeur diagnostiquée.

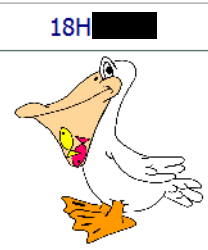

- Rechercher un Pélican

Par le numéro du prélèvement :

Par la référence de l'extemporané :

Par une partie de la conclusion

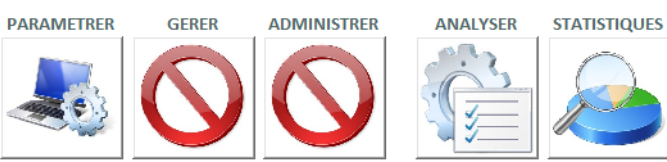

V Afficher les avertissements de circulation des Pélicans

\section{B}

\section{INFORMATIONS COMMUNES A TOUS LES PRELEVEMENTS}

- Identification du Compte Rendu ( Prélèvement)

NNo d'identification : $\quad 18 \mathrm{H}$

Date du Prélèvement: $\longdiv { 0 4 / 0 6 / 2 0 1 8 }$ Heure :

Renseignements cliniques (compte rendu opératoire)

Méningiome sphéno-orbitaire gauche.

Informations sur le prélèvement

$\odot$ Biopsie

$\Gamma$ Examen extemporané
- Résection chirurgicale

$\Gamma$ Autre :
Praticiens concernés

Chirurgien :

Anatomopathologiste : GAUCHOTTE Guillaume (Pr)

\section{Description macroscopique}

Quatre fragments de consistance molle, blanchâtres et friables, mesurant de 1 à $3 \mathrm{~cm}$ de grand axe.

Qualité du prélèvement :

$$
\begin{aligned}
& \odot \text { Interprétable } \bigcirc \text { Difficilement Interprétable } \\
& \bigcirc \text { Non Interprétable }
\end{aligned}
$$

Classification oms de la Tumeur

Groupe d'appartenance de la Tumeur:

Adénomes (Tumeurs neuro-endocrines OMS 2017) _ Autres gliomes

Autres tumeurs astrocytaires

Lymphomes

Lymphomes (Non répertoriés OMS 2016)
Données spécifiques

Conclusion

$-$ 
A

\section{CANCER : Système nerveux central - GROUPE DE TUMEURS : Méningiomes}

Examen du prélèvement |Analyses

\begin{tabular}{|c|c|c|c|}
\hline Convexité & $\odot$ ouI & - NON & $\triangle$ Non Renseigné \\
\hline Base du crâne & • OUI & $C$ NON & $\bigcirc$ Non Renseigné \\
\hline Intraventriculaire & $C$ OUI & $C$ NON & - Non Renseigné \\
\hline Spinal & C OUI & $C$ NON & - Non Renseigné \\
\hline Autre & $C$ OUI & C NON & - Non Renseigné \\
\hline
\end{tabular}

\section{--- Architecture et contingent cellulaire d'intérêt pronostique}

Diffuse

Chordoïde

$C$ OUI $C$ NON

$C$ DIFFUS C FOCAL $\odot$ NON

$C$ DIFFUS $C$ FOCAL $\bullet$ NON

$\checkmark$ DIFFUS $\triangle$ FOCAL $\bullet$ NON

$C$ DIFFUS $C$ FOCAL $\odot$ NON

$C$ OUI C NON

$\triangle$ OUI $C$ NON

- Non Renseigné

$\checkmark$ Non Renseigné

Non Renseigné

$\checkmark$ Non Renseigné

$C$ Non Renseigne

c Non Renseigné

• Non Renseigné

Travées collagéniques

$C$ Non Renseigné Mitoses

- Nombre de mitoses pour 10 CFG / $1, \ldots$

Infiltration du parenchyme cérébral

- OUI C NON

$1 / 1,6 \mathrm{~mm}^{2}$

C OUI $\odot$ NON

Non Renseigné

--- Critères histopronostiques morphologiques mineurs

Hypercellularite

Nécrose tumorale

$C$ OUI $\odot$ NON

$r$ OUI $\bullet$ NON

Non Renseigné

Petites cellules (avec rapport N/C élevé)

$c$ OUI $\bullet$ nON

$C$ OUI $\bullet$ NON

Perte de l'architecture tumorale C OUI C NON

$C$ Non Renseigne

$\checkmark$ Non Renseigné

$C$ Non Renseigné

$C$ Non Renseigné

Infiltration dure-mère

Infiltration osseuse

Prolifération endothéliocapillaire

Hyperplasie endothéliale

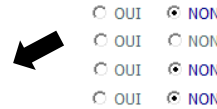

$C$ OUI - NON

Non Renseigné

- Non Renseigné

$\checkmark$ Non Renseigné

Non Renseigné
B

\section{CANCER : Système nerveux central - GROUPE DE TUMEURS : Méningiomes}

Examen du prélèvement Analyses

--- Immunohistochimie

EMA

ACE

NCAM

Récepteurs à la progestérone

Valeur Récepteurs à la progestérone

Ki67

- Valeur Ki67

CD34

ALDH1

STAT6

SSTR2

$\mathrm{BCl} 2$

Autres techniques $\checkmark$ NON FAIT $C$ NEGATIF $C$ POSITIF $\bullet$ Non Renseigné

$\checkmark$ NON FAIT $C$ NEGATIF $C$ POSITIF $\bullet$ Non Renseigne

$C$ NON FAIT $C$ NEGATIF $C$ POSITIF $\bullet$ Non Renseigne

$\triangle$ NON FAIT $\triangle$ NEGATIF $\odot$ POSITIF $C$ Non Renseigné $90 \%$

$C$ NON FAIT $C$ NEGATIF $\odot$ POSITIF $C$ Non Renseigné $2 \%$

$\checkmark$ NON FAIT $\subset$ NEGATIF $C$ POSITIF $\bullet$ Non Renseigne

$\checkmark$ NON FAIT $\subset$ NEGATIF $\subset$ POSITIF $\bullet$ Non Renseigne

$\checkmark$ NON FAIT $\subset$ NEGATIF $\subset$ POSITIF $\bullet$ Non Renseigné

$\backsim$ NON FAIT $\backsim$ NEGATIF $\backsim$ POSITIF $\odot$ Non Renseigne

$\checkmark$ NON FAIT $C$ NEGATIF $C$ POSITIF $\bullet$ Non Renseigné

$\triangle$ OUI $C$ NON

- Non Renseigne

C

- Classification OMS de la Tumeur

CANCER : Système nerveux central

GROUPE : Méningiomes

SNOMED: 9531/0

Grade Histopronostique

Libellé de la Tumeur diagnostiquée :

ADICAP : NOK2

Méningiome (sans autre indication)

Méningiome à cellules claires

Méningiome à stroma lymphoïde

Méningiome anaplasique

Méningiome angiomateux

Méningiome atypique

Méningiome chordoïde

Méningiome fibroblastique

Méningiome méningothélia

Méningiome métaplasique (chondroïde et osseux)

Meningothelial meningioma

- Conclusion (Diagnostic)

Méningiome méningothélial. 
Tableau 1. Descriptif général des principales données concernant les méningiomes extraites à partir de l'application PELICAN. General data about meningioma, after exportation from the PELICAN software.

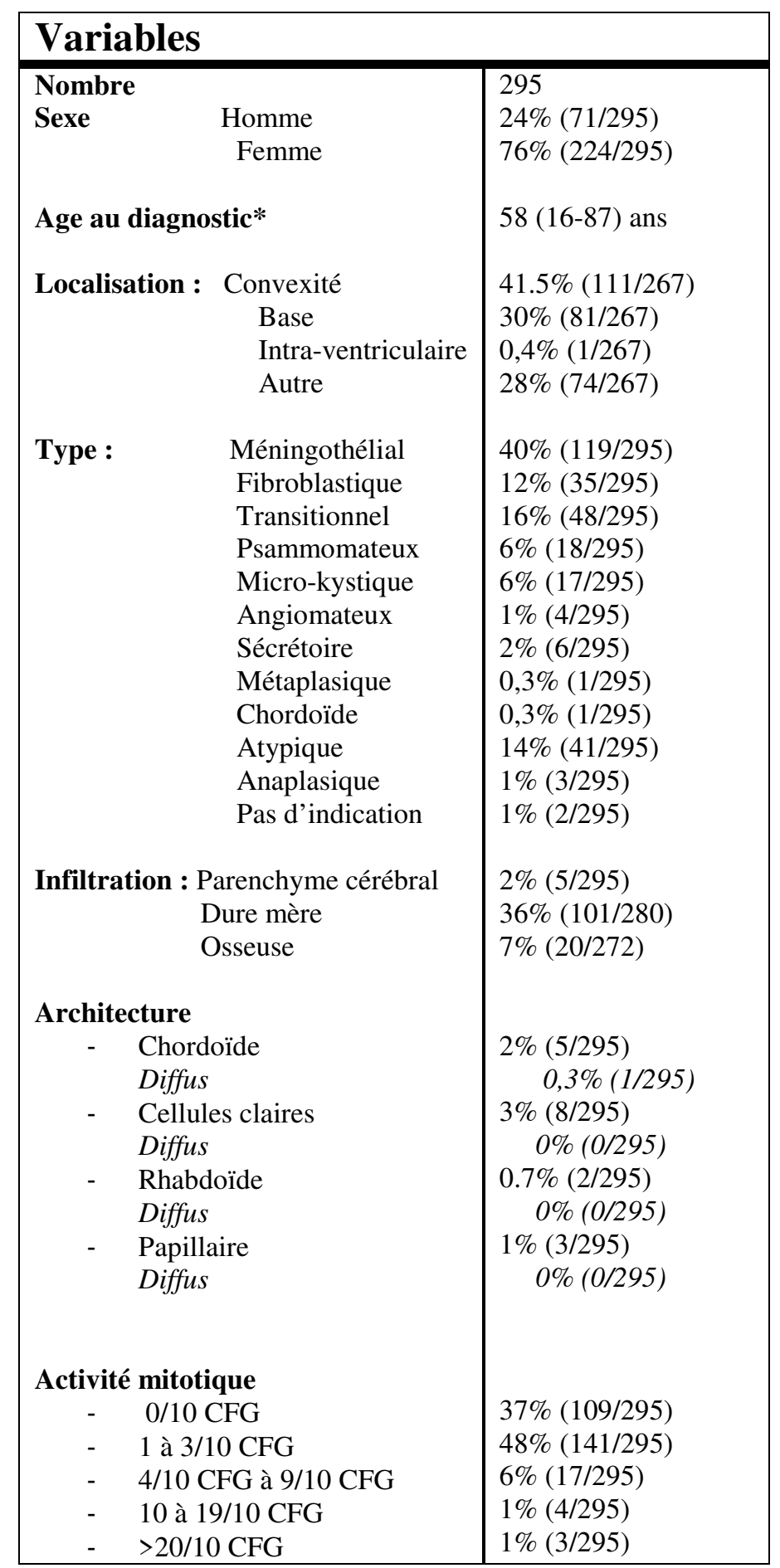

moy. : moyenne ; min. : minimum ; max. maximum ; CFG : champ au fort grossissement $\left(0,16 \mathrm{~mm}^{2}\right)$ 
Tableau 2. Comparaison des données cliniques, histologiques et immunohistochimiques entre les méningiomes de grade I et les méningiomes de grade II (test du Khi² ou de Fisher pour les données qualitatives, test de Mann-Whitney-Wilcoxon pour les données quantitatives).

Comparison of clinical, histological and immunohistochemical data between grade I and grade II meningiomas (Khi or Fisher test for qualitative variables, Mann-Whitney-Wilcoxon test for quantitative variables).

\begin{tabular}{|c|c|c|c|c|}
\hline \multicolumn{2}{|l|}{ Variables } & $\begin{array}{l}\text { Méningiomes } \\
\text { de grade I }\end{array}$ & $\begin{array}{l}\text { Méningiomes de } \\
\text { grade II }\end{array}$ & p-value \\
\hline \multicolumn{2}{|l|}{ Nombre } & 250 & 42 & \\
\hline \multicolumn{2}{|c|}{ Age (moy. ; min.-max.) } & 58 ans $(16-87)$ & 60 ans $(27-85)$ & $\mathrm{p}=0,54$ \\
\hline \multirow{2}{*}{\multicolumn{2}{|c|}{ Sexe }} & Homme $(53 / 250)$ & Homme (18/42) & $\mathrm{p}=0,002^{*}$ \\
\hline & & Femme $(197 / 250)$ & Femme $(24 / 42)$ & \\
\hline \multicolumn{2}{|c|}{$\begin{array}{l}\text { Antécédent personnel de tumeur du système } \\
\text { nerveux central, phacomatose ou de cancer }\end{array}$} & $23 \%(25 / 110)$ & $49 \%(17 / 35)$ & $\mathrm{p}=0,003^{*}$ \\
\hline \multicolumn{5}{|l|}{ Symptômes } \\
\hline \multicolumn{2}{|c|}{ - Asymptomatique } & $0 \%(0 / 91)$ & $7 \%(2 / 30)$ & $\mathrm{p}=0,06$ \\
\hline \multicolumn{2}{|c|}{ - Epilepsie } & $19 \%(17 / 91)$ & $20 \%(6 / 30)$ & $\mathrm{p}=1$ \\
\hline \multicolumn{2}{|c|}{ - Céphalée } & $27 \%(25 / 91)$ & $20 \%(6 / 30)$ & $p=0,47$ \\
\hline \multicolumn{2}{|c|}{ - Hypertension intra-crânienne } & $0 \%(0 / 91)$ & $0 \%(0 / 30)$ & $\mathrm{p}=1$ \\
\hline \multicolumn{2}{|c|}{ - Troubles des fonctions supérieures } & $35 \%(32 / 91)$ & $37 \%(11 / 30)$ & $\mathrm{p}=1$ \\
\hline \multicolumn{2}{|c|}{ - Déficit neurologiques } & $3 \%(3 / 91)$ & $13 \%(4 / 30)$ & $\mathrm{p}=0,06$ \\
\hline \multicolumn{2}{|c|}{ - $\quad$ Autres } & $31 \%(28 / 91)$ & $33 \%(10 / 30)$ & $\mathrm{p}=1$ \\
\hline \multicolumn{2}{|l|}{ Score OMS } & $0(100 \% ; n=217)$ & $0(100 \% ; n=23)$ & $\mathrm{p}=1$ \\
\hline \multicolumn{2}{|c|}{ Délai entre les symptômes et la chirurgie : } & & & $\mathrm{p}=1$ \\
\hline \multicolumn{2}{|c|}{ - $\quad 1$ à 12 mois } & $92 \%(201 / 218)$ & $95 \%(23 / 24)$ & \\
\hline \multicolumn{2}{|c|}{ - $\quad 12$ à 24 mois } & $5 \%(11 / 218)$ & $5 \%(1 / 24)$ & \\
\hline \multicolumn{2}{|c|}{$-\quad>24$ mois } & $3 \%(6 / 218)$ & $0 \%(0 / 24)$ & \\
\hline \multicolumn{5}{|c|}{ Critères histo-pronostiques } \\
\hline \multicolumn{2}{|c|}{ - Hyper-cellularité } & $7 \%(17 / 250)$ & $57 \%(27 / 42)$ & \\
\hline \multicolumn{2}{|c|}{ Nécrose } & $6 \%(15 / 250)$ & $71 \%(30 / 42)$ & \\
\hline \multicolumn{2}{|c|}{ - $\quad$ Petites cellules } & $8 \%(19 / 250)$ & $50 \%(21 / 42)$ & \\
\hline \multicolumn{2}{|c|}{ - Nucléole proéminent } & $12 \%(29 / 250)$ & $59.5 \%(25 / 42)$ & \\
\hline \multirow{2}{*}{\multicolumn{2}{|c|}{-}} & $12 \%(30 / 250)$ & $55 \%(23 / 42)$ & \\
\hline & & $0 \%(0 / 250)$ & $71 \%(30 / 42)$ & \\
\hline \multicolumn{2}{|c|}{ Hyperplasie endothéliale } & $12 \%(3 / 26)$ & $75 \%(6 / 8)$ & $\mathrm{p}=0,002 *$ \\
\hline \multicolumn{2}{|c|}{ Prolifération endothélio-capillaire } & $8 \%(2 / 26)$ & $50 \%(4 / 8)$ & $\mathrm{p}=0,03^{*}$ \\
\hline \multirow[t]{3}{*}{ Infiltration : } & Parenchyme cérébral & $0 \%(0 / 250)$ & $10 \%(4 / 42)$ & $\mathrm{p}<0,0001^{*}$ \\
\hline & Dure mère & $31 \%(74 / 236)$ & $64 \%(26 / 41)$ & $\mathrm{p}<0,001 *$ \\
\hline & Osseuse & $7 \%(15 / 229)$ & $13 \%(5 / 40)$ & $\mathrm{p}=0,32$ \\
\hline \multirow{4}{*}{\multicolumn{2}{|c|}{$\begin{array}{l}\text { Indice mitotique } / 1,6 \mathbf{~ m m}^{2} \text { (moy. ; min.-max.) } \\
\text { Indice mitotique }<4 / 1.6 \mathbf{m m}^{2} \text { (moy. ; min.-max.) } \\
\text { Ki-67 (moy. ; min.-max.) } \\
\text { Récepteurs de la progestérone (moy. ; min.-max.) }\end{array}$}} & $0,72(0-3)$ & $4,52(0-16)$ & $\mathrm{p}<0,001^{*}$ \\
\hline & & $100 \%(250 / 250)$ & $50 \%(21 / 42)$ & \\
\hline & & $5 \% ; 0-80 \%$ & $16 \% ; 2-50 \%$ & $\mathrm{p}<0,0001 *$ \\
\hline & & $48 \% ; 0-100 \%$ & $28 \% ; 0-100 \%$ & $\mathrm{p}<0,001 *$ \\
\hline
\end{tabular}

moy. : moyenne ; min. : minimum ; max. maximum ; OMS : Organisation Mondiale de la Santé ; ${ }^{*}$ résultat statistiquement significatif $(\mathrm{p}<0.05)$ 
Tableau 3. Profil immunohistochimique des méningiomes selon leur sous-type histologique. Immunohistochemical profil of meningiomas according to histological subtype.

\begin{tabular}{|l|lllll|}
\hline \multicolumn{1}{|l}{ Type/IHC } & EMA & RP & CD34 & STAT6 & SSTR2 \\
\hline Méningothélial & $89 \%(17 / 19)$ & $95 \%(97 / 102)$ & $0 \%(0 / 1)$ & $0 \%(0 / 5)$ & $97,5 \%(39 / 40)$ \\
Fibroblastique & $87, .5 \%(7 / 8)$ & $87,5 \%(28 / 32)$ & $0 \%(0 / 5)$ & $0 \%(0 / 23)$ & $96 \%(23 / 24)$ \\
Pransitionnel & N.R. & $100 \%(47 / 47)$ & $0 \%(0 / 1)$ & $0 \%(0 / 3)$ & $100 \%(15 / 15)$ \\
Micrommomateux & $100 \%(3 / 3)$ & $100 \%(15 / 15)$ & N.R. & N.R. & $100 \%(5 / 5)$ \\
Angiomateux & $100 \%(2 / 2)$ & $100 \%(4 / 4)$ & $0 \%(0 / 1)$ & $0 \%(0 / 3)$ & $100 \%(3 / 3)$ \\
Sécrétoire & $100 \%(1 / 1)$ & $100 \%(4 / 4)$ & N.R. & N.R. & N.R. \\
Métaplasique & N.R. & $100 \%(1 / 1)$ & N.R. & N.R. & N.R. \\
\hline
\end{tabular}

IHC : immunohistochimie ; N.R. : non renseigné. 